\title{
The normalization of wave functions of the continuous spectrum
}

\author{
M. Amaku ${ }^{1,2 @}$, F. A. B. Coutinho ${ }^{*}$, F. M. Toyama ${ }^{3}$ \\ ${ }^{1}$ Universidade de São Paulo, Faculdade de Medicina, São Paulo, SP, Brasil \\ ${ }^{2}$ Universidade de São Paulo, Faculdade de Medicina Veterinária e Zootecnia, São Paulo, SP, Brasil \\ ${ }^{3}$ Kyoto Sangyo University, Department of Computer Science, Kyoto 603-8555, Japan
}

Received on April 30, 2019; Revised on June 28, 2019; Accepted on August 17, 2019.

\begin{abstract}
The continuous spectrum of a quantum mechanical (QM) system contains important information on the system. However, the normalization of wave functions of the continuous spectrum is often difficult and therefore is often omitted from the books on QM. We discuss this problem and show how we can overcome this problem technically, with some examples.
\end{abstract}

Keywords: continuous spectrum, normalization, distribution theory

\section{Introduction}

A physical system in quantum mechanics is characterized by its Hamiltonian, which is a self-adjoint operator in a Hilbert Space. Assume for now that the system is one dimensional. Then the Hilbert space are complex functions of a real variable $\psi(x)$, such that

$$
\int_{-\infty}^{\infty} \psi^{*}(x) \psi(x) d x=A \text { (finite) }
$$

The symbol * stands for complex conjugate. The functions $\psi(x)$ are the states of the systems. If we define

$$
\phi(x)=\frac{1}{A^{1 / 2}} \psi(x),
$$

we say that we have normalized the wave function and we have

$$
\int_{-\infty}^{\infty} \phi^{*}(x) \phi(x) d x=1
$$

Remark 1 In three dimensions, for central potentials, the differential equations for the radial part of the eigenfunction is identical to the equation for one dimension except by the centrifugal barrier. Also, the integrations in equations (1) and (3) are from 0 to $\infty$.

Let $u_{E}(x)$ be an eigenfunction of a Hamiltonian $H$, i.e.,

$$
H u_{E}(x)=E u_{E}(x) .
$$

Sometimes, only some (or none) of the eigenfunctions are normalizable in the usual sense, the others (or all of them) are such that

$$
\int_{-\infty}^{\infty} u_{E}^{*}(x) u_{E}(x) d x=\infty
$$

*Correspondence email address: coutinho@dim.fm.usp.br
The values of $E$ for such eigenfunctions form a continuum interval, as we shall see. The eigenfunctions $u_{E}(x)$ are said to be eigenfunctions of the continuous spectrum of the Hamiltonian operator.

A very important property of the Hamiltonian is that it has a complete set of eigenfunctions. They form a complete set of functions in the sense that any state of the system can be expanded in a series, integrals or a mixture of both series and integrals of these functions, that is,

$$
\psi(x)=\sum c_{i} u_{E_{i}}(x)+\int \phi(E) u_{E}(x) d E .
$$

where

$$
c_{i}=\int u_{E_{i}}^{*}(x) \psi(x) d x
$$

and

$$
\phi(E)=\int u_{E}^{*}(x) \psi(x) d x .
$$

However, $u_{E_{i}}$ and $u_{E}(x)$ must be properly normalized. The summation over the bound states $i$ (equation (7) ) and the values of $E$ in equation $(8)$ depend on the system under study, as we shall see later.

The eigenfunctions on the second term of the equation (6) belong to the continuous spectrum and it is important that the eigenfunctions be normalized, in a special way, as we will explain below.

\section{The location of the continuous spectrum and its normalization}

Consider the problem of a particle in one dimension subjected to a potential that has the form shown in Figure 1. 


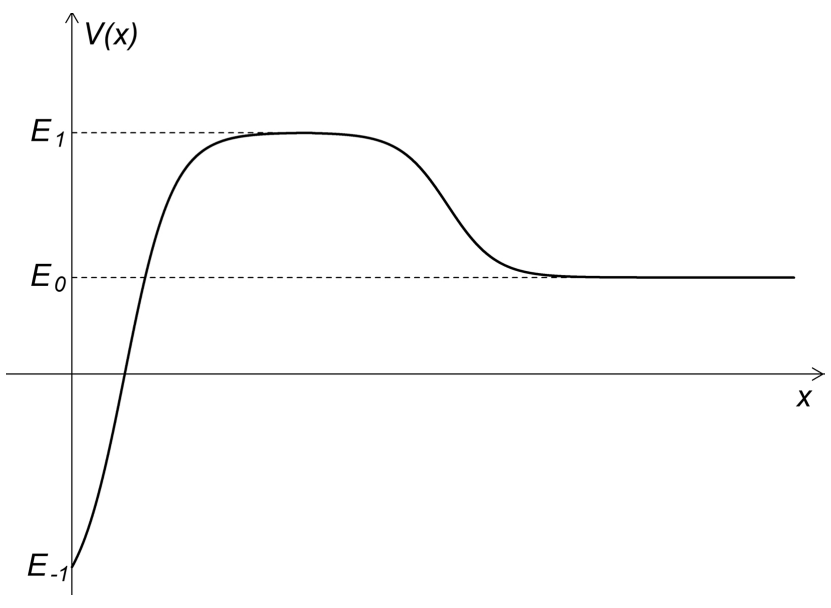

Figure 1: A potential with possible bound states (between $E_{-1}$ and $E_{0}$ ) and continuous spectrum for $E>E_{0}$ (with possible resonances between $E_{0}$ and $E_{1}$ ).

Almost all books on quantum mechanics show that for energies between $E_{-1}$ and $E_{0}$ there exist eigenfunctions that are bound states, whose wave functions $\psi_{E_{i}}(x)$ are normalizable, that is,

$$
\int d x\left|u_{E_{i}}(x)\right|^{2}=\text { finite. }
$$

If we have two or more normalizable states with the same energy $E_{i}=E_{j}$ we can arrange things so the

$$
\int d x u_{E_{i}}^{*}(x) u_{E_{j}}(x)=\delta_{i j} .
$$

In the above formula, $\delta_{i j}$ is 1 if $i=j$ and zero otherwise.

For energies greater than $E_{0}$, the eigenfunctions are not normalizable as in equation (2) because these wave functions $u_{E}(x)$ are such that

$$
\left\|u_{E}(x)\right\|=\int d x u_{E}^{*}(x) u_{E}(x)=\infty .
$$

These eigenfunctions are said to be of the continuous spectrum of the Hamiltonian that in the above case extends from $E_{0}$ to $\infty$.

Figure 2 shows an even more interesting situation. In this case, the potential is given by 1

$$
V(x)= \begin{cases}-V_{0}-F x, & 0<x<a, \\ -F x, & x>a .\end{cases}
$$

In this case, the system have no normalizable eigenfunctions and the spectrum is continuous from $-\infty<E<\infty$.

For the eigenfunctions of the continuous spectrum to be useful, however, we have to "normalize" them in a special way. It is possible to proof [2, p. 99] that, when correctly normalized, any eigenfunction of the continuous spectrum, say $u_{E}(x)$, is such that

$$
\left\|u_{E}(x) u_{E^{\prime}}(x)\right\|=\int u_{E^{\prime}}^{*}(x) u_{E}(x) d x=\delta\left(E-E^{\prime}\right),
$$

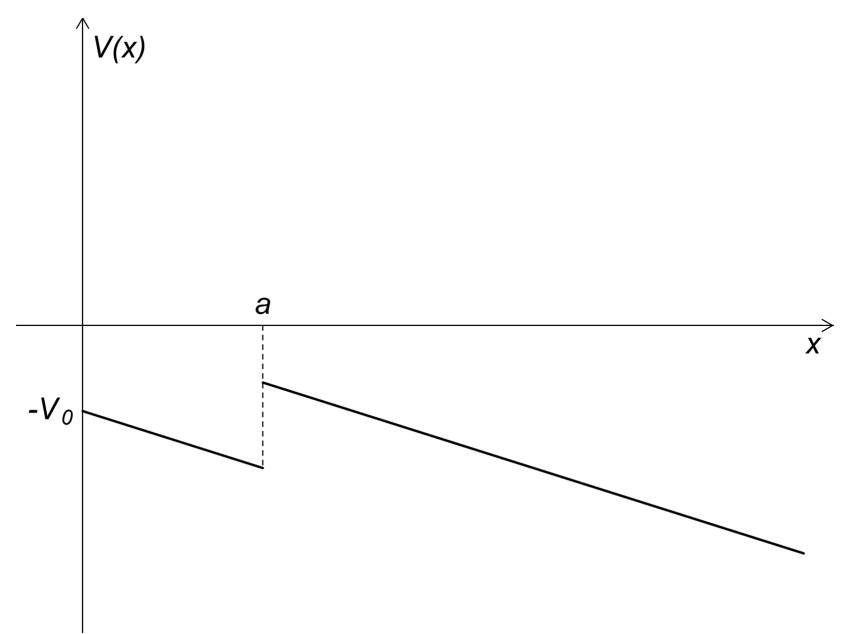

Figure 2: Graph of the potential given by equation 12 .

where $\delta$ is the Dirac delta function. However, as stressed above, one has to correctly normalize the $u_{E}(r)$. This involves the difficult evaluation of divergent integrals to show that the resulting mathematical objects are $\delta$ functions 3, p. 237] [4,5], that is, that in fact they obey equation 13 .

The purpose of this article is to show ways of performing these difficult calculations. In the next section, we show three methods of normalizing the wave functions of the continuous spectrum.

\section{Methods of normalizing the wave functions of the continuous spectrum}

\subsection{Calculating divergent integrals}

This is, in general, the most difficult method and should be used parsimoniously. It should be noted that divergent integrals sometimes produces distributions but they are not always the Dirac delta function distribution [6, p. 71], that is, they do not obey equation (13). We shall elaborate below about how to proof that a certain normalization results in a delta function.

Example 1) Normalize the following eigenfuntions of the continuous spectrum of the following problem 7

Consider the Hamiltonian $H=-\frac{d^{2}}{d x^{2}}$ defined for $0<$ $x<\infty$ with boundary condition $\phi(0)=0$. The solution of the equation $H \phi=E \phi$ is $\phi=A \sin (k x)$ where $k^{2}=E$. To normalize $\phi$ we have to use the relation

$$
\int_{0}^{\infty} \sin \left(k_{1} x\right) \sin \left(k_{2} x\right) d x=\frac{\pi}{2} \delta\left(k_{1}-k_{2}\right), k_{1}, k_{2}>0
$$

so that $A=\left(\frac{2}{\pi}\right)^{1 / 2}$.

To prove relation (14) we show that it obeys the following defining property of the Dirac Delta function, 
namely:

$$
\int_{0}^{\infty} f\left(k_{1}\right) \delta\left(k_{1}-k_{2}\right) d k_{1}=f\left(k_{2}\right)
$$

This property is actually a rigorous definition of the Dirac delta function. If the reader feels uncomfortable with this definition, he/she should read Remark 2 for clarification.

To demonstrate 14 we calculate

$$
\begin{aligned}
& \lim _{L \rightarrow \infty} \int_{0}^{\infty} f\left(k_{1}\right) d k_{1} \int_{0}^{L} \sin \left(k_{1} x\right) \sin \left(k_{2} x\right) d x= \\
& =\frac{1}{2} \pi f\left(k_{2}\right) .
\end{aligned}
$$

The details of this calculation are presented in Brownstein [7] and repeated here for completeness and also because there are a few misprints in his article

$$
\begin{aligned}
& \lim _{L \rightarrow \infty} \int_{0}^{\infty} f\left(k_{1}\right) d k_{1} \int_{0}^{L} \sin \left(k_{1} x\right) \sin \left(k_{2} x\right) d x= \\
& =\lim _{L \rightarrow \infty} \frac{1}{2} \int_{0}^{\infty} f\left(k_{1}\right) d k_{1} \\
& \times\left(\frac{\sin \left[\left(k_{1}-k_{2}\right) L\right]}{k_{1}-k_{2}}-\frac{\sin \left[\left(k_{1}+k_{2}\right) L\right]}{k_{1}+k_{2}}\right) .
\end{aligned}
$$

Consider the first integral and change variable

$$
\frac{\xi}{L}=\left(k_{1}-k_{2}\right)
$$

Then the first integral becomes

$$
\begin{aligned}
& \lim _{L \rightarrow \infty} \frac{1}{2} \int_{-k_{2} L}^{\infty} \frac{\frac{f\left(k_{2}+\frac{\xi}{L}\right) d \xi}{L}}{\frac{\xi}{L}} \sin \xi \\
& =\frac{1}{2} f\left(k_{2}\right)\left(\int_{-\infty}^{0} \frac{\sin \xi}{\xi} d \xi+\int_{0}^{\infty} \frac{\sin \xi}{\xi} d \xi\right) \\
& =f\left(k_{2}\right) \int_{0}^{\infty} \frac{\sin \xi}{\xi} d \xi \\
& =\frac{\pi}{2} f\left(k_{2}\right) .
\end{aligned}
$$

We have used (see [8, p. 285])

$$
\int_{0}^{\infty} \frac{\sin x}{x} d x=\frac{\pi}{2}
$$

The second integral gives zero in the limit $L \rightarrow \infty$, as can be easily verified. In fact, the second integral after the transformation

$$
\frac{\xi}{L}=\left(k_{1}+k_{2}\right)
$$

becomes

$$
-\lim _{L \rightarrow \infty} \frac{1}{2} \int_{k_{2} L}^{\infty} \frac{\frac{f\left(-k_{2}+\frac{\xi}{L}\right) d \xi}{L}}{\frac{\xi}{L}} \sin \xi
$$

that vanishes because the lower limit of integration approaches $\infty$ as $L \rightarrow \infty$.

Example 2) Normalize the following eigenfunction of the continuous spectrum of the following problem

Consider the Hamiltonian $-\frac{d^{2}}{d x^{2}}$ defined for $0<x<\infty$ with boundary condition $\phi^{\prime}(0)=0$. The eigenfunctions of the problem $H \phi(x)=E \phi(x)$ are $\phi(x)=B \cos (k x)$ where $k^{2}=E$.

To normalize $\phi(x)$ we use the relation

$$
\int_{0}^{\infty} \cos \left(k_{1} x\right) \cos \left(k_{2} x\right) d x=\frac{\pi}{2} \delta\left(k_{1}-k_{2}\right)
$$

and hence $B=\left(\frac{2}{\pi}\right)^{1 / 2}$.

The proof of this relation is identical to the proof of relation 16 .

Example 3) Normalize the following eigenfuntions of the continuous spectrum

Consider the Hamiltonian $-\frac{d^{2}}{d x^{2}}$ defined for $0<x<\infty$ with boundary condition $\psi^{\prime}(0)=\alpha \psi(0)$. The eigenfunctions of this problem are

$$
\psi(x)=\cos (k x)+\frac{\alpha}{k} \sin (k x)
$$

where $k^{2}=E$.

To normalize this function we need the relations (14), (23) and the following relation

$\int_{0}^{\infty}\left[k_{2} \sin \left(k_{1} x\right) \cos \left(k_{2} x\right)+k_{1} \sin \left(k_{2} x\right) \cos \left(k_{1} x\right)\right] d x=0$. (25)

To prove equation 25 we replace the upper limit of the integral by $L$, multiply by a function $f\left(k_{1}\right)$ and integrate to get

$$
\begin{aligned}
& \lim _{L \rightarrow \infty} \int_{0}^{\infty} f\left(k_{1}\right) d k_{1} \int_{0}^{L}\left[k_{2} \sin \left(k_{1} x\right) \cos \left(k_{2} x\right)\right. \\
& \left.+k_{1} \sin \left(k_{2} x\right) \cos \left(k_{1} x\right)\right] d x= \\
& \lim _{L \rightarrow \infty} \int_{0}^{\infty} f\left(k_{1}\right) d k_{1} \int_{0}^{L} \frac{d}{d x}\left[\sin \left(k_{1} x\right) \sin \left(k_{2} x\right) d x\right]= \\
& \lim _{L \rightarrow \infty} \int_{0}^{\infty} f\left(k_{1}\right) d k_{1} \sin \left(k_{1} L\right) \sin \left(k_{2} L\right)=0,
\end{aligned}
$$

where we have used the relation (see [9, p. 310])

$$
\lim _{L \rightarrow \infty} \int_{0}^{\infty} f\left(k_{1}\right) \sin \left(k_{1} L\right) d k_{1}=0
$$

After some more calculations we get the properly normalized eigenfunction which is

$$
\psi(x)=\left(\frac{2}{\pi}\right)^{\frac{1}{2}} k\left(\alpha^{2}+k^{2}\right)^{-\frac{1}{2}}\left(\cos (k x)+\frac{\alpha}{k} \sin (k x)\right) .
$$

We shall give another demonstration of this formula in the example 5 . 


\subsection{Normalizing the eigenfunctions when} $V(x) \rightarrow 0$ sufficiently fast as $x \rightarrow \infty$

We first examine the equation that results for $V(x) \equiv 0$

$$
-\frac{d^{2}}{d x^{2}} \phi(x)=E \phi(x)
$$

with boundary conditions $\phi(0)=0$ and $\phi^{\prime}(0)=1$.

We have only eigenfunctions of the continuous so that we can write equations (6) and (8) as

$$
\begin{aligned}
& \psi(x)=\int_{0}^{\infty} \phi(E) u_{E}(x) d E \\
& \phi(E)=\int_{0}^{\infty} u_{E}(x) \psi(x) d x .
\end{aligned}
$$

But since $u_{E}(x)=\frac{\sin \sqrt{E} x}{\sqrt{E}}$ we have

$$
\phi(E)=\int_{0}^{\infty} \frac{\sin \sqrt{E} x}{\sqrt{E}} \psi(x) d x
$$

and

$$
\psi(x)=\int_{0}^{\infty} \phi(E) \frac{\sin \sqrt{E} x}{\sqrt{E}} d E .
$$

The correct normalization of a eigenfunction of the continuous spectrum of a problem such that $V(x) \rightarrow 0$ as $x \rightarrow \infty$ is obtained by matching the eigenfunction to equation (33) when $x \rightarrow \infty$ [1].

\section{Example 4) A square well}

Consider the case when $V(x)$ is given by $V(x)=-V_{0}$ , $\left(V_{0}>0\right)$ for $0<x<a$ and $V(x)=0$ for $x>a$ with boundary condition $\psi(0)=0$ (an impenetrable barrier to the left).

The eigenfuction for $E>0$ is given by

$$
\psi_{k}(x)=A(k) \sin (K x) \quad \text { for } x<a
$$

and

$$
\begin{aligned}
\psi_{k}(x) & =B(k)(\sin \delta \cos k x+\cos \delta \sin k x) \\
& =B(k) \sin (k x+\delta(k)) \quad \text { for } x>a,
\end{aligned}
$$

where $K=\left(E+V_{0}\right)^{1 / 2}$ and $k=E^{1 / 2}$.

The ratio $B(k) / A(k)=C(k)$ can be determined by imposing continuity of the eigenfunction at $x=a$ and by imposing continuity of the derivative of the eigenfunction at $x=a$.

The wave function will be properly normalized by imposing the condition $C(k)=(2 / \pi)^{1 / 2}$.

To proof that this recipe produces the proper normalized eigenvalue we proceed as follows:

Consider the eigenfunction at two values of the energy $k_{1}$ and $k_{2}$. Then using the Schrodinger equation we get
At $x=0$ the left hand side of the previous equation gives zero and using the asymptotic value of the eigenfunctions, namely $C\left(k_{1}\right) \sin \left(k_{1} x+\delta_{1}\right)$ and $C\left(k_{2}\right) \sin \left(k_{2} x+\delta_{2}\right)$, we get

$$
\begin{aligned}
& \int_{0}^{R} \psi_{k_{1}}^{*} \psi_{k_{2}} d x=\frac{1}{2}\left(C\left(k_{1}\right) C\left(k_{2}\right)\right) \\
& \times\left\{\frac{1}{k_{1}-k_{2}} \sin \left[\left(k_{1}-k_{2}\right) R+\delta_{1}-\delta_{2}\right]\right. \\
& \left.-\frac{1}{k_{1}+k_{2}} \sin \left[\left(k_{1}+k_{2}\right) R+\delta_{1}+\delta_{2}\right]\right\} .
\end{aligned}
$$

Now, we want to show that the mathematical object $\int_{0}^{R} \psi_{k_{1}}^{*} \psi_{k_{2}} d x$ is proportional to $\delta\left(k_{1}-k_{2}\right)$ as $R \rightarrow \infty$. To do this, we have to show that this object multiplied by any good function $f\left(k_{2}\right)$ and integrated in $R$ in this limit gives $f\left(k_{1}\right)$.

The second part

$$
\begin{aligned}
& \int_{0}^{R}-\frac{1}{2}\left(C\left(k_{1}\right) C\left(k_{2}\right)\right)\left\{\frac{1}{k_{1}+k_{2}}\right. \\
& \left.\times \sin \left[\left(k_{1}+k_{2}\right) R+\delta_{1}+\delta_{2}\right]\right\} f\left(k_{2}\right) d R
\end{aligned}
$$

gives zero because the term between brackets oscillates violently as $R \rightarrow \infty$.

The first part

$$
\begin{aligned}
& \int_{0}^{R}-\frac{1}{2}\left(C\left(k_{1}\right) C\left(k_{2}\right)\right)\left\{\frac{1}{k_{1}-k_{2}}\right. \\
& \left.\times \sin \left[\left(k_{1}-k_{2}\right) R+\delta_{1}-\delta_{2}\right]\right\} f\left(k_{2}\right) d R
\end{aligned}
$$

also goes to zero if $k_{1} \neq k_{2}$. However when $k_{1} \rightarrow k_{2}, \delta_{1}$ cancels with $\delta_{2}$ and we are left with

$$
\begin{aligned}
& \int_{0}^{R}-\frac{1}{2}\left(C\left(k_{1}\right) C\left(k_{2}\right)\right)\left\{\frac{1}{k_{1}-k_{2}} \sin \left[\left(k_{1}-k_{2}\right) R\right]\right\} \\
& f\left(k_{2}\right) d R .
\end{aligned}
$$

Therefore, in the limit as $R \rightarrow \infty$, we get

$$
\begin{aligned}
& \lim _{R \rightarrow \infty} \int_{0}^{R}-\frac{1}{2}\left(C\left(k_{1}\right) C\left(k_{2}\right)\right)\left\{\frac{1}{k_{1}-k_{2}} \sin \left[\left(k_{1}-k_{2}\right) R\right]\right\} \\
& f\left(k_{2}\right) d R=\frac{\pi}{2}\left|C\left(k_{1}\right)\right|^{2} \delta\left(k_{1}-k_{2}\right) f\left(k_{1}\right),
\end{aligned}
$$

as we showed in equation 16 above. A different approach to this problem is shown in Remark 2.

Hence the proper normalization is obtained by making $|C(k)|=(2 / \pi)^{1 / 2}$ as claimed. The eigenfunctions for $E>0$ are then given by

$$
\psi_{k}(x)=\left(\frac{2}{\pi}\right)^{\frac{1}{2}} C(k)^{-1} \sin (K x), \quad \text { for } x<a
$$

and

$$
\begin{aligned}
& \psi_{k}(x)=\left(\frac{2}{\pi}\right)^{\frac{1}{2}}(\sin \delta \cos k x+\cos \delta \sin k x) \\
& =\left(\frac{2}{\pi}\right)^{\frac{1}{2}} \sin (k x+\delta(k)), \quad \text { for } x>a,
\end{aligned}
$$


where $K=\left(E+V_{0}\right)^{1 / 2}$ and $k=E^{1 / 2}$.

The above calculation was adapted from the book by Perelomov and Zel'dovich [10, p. 53-55]. It is however more convenient to use the following result, that can be found in [3, p. 247] and that is based in the following theorem:

Theorem 1 (Friedman [3]) The eigenfunctions of the continuous spectrum $u_{k}(x)$ of the operator $-\frac{d^{2}}{d x^{2}}+V(x)$, where $V(x)$ vanishes when $x \rightarrow \infty$ and $u_{k}(x)$ satisfies some boundary condition at $x=0$ are properly normalized if they are solutions of the equation

$$
\frac{d^{2} u_{k}(x)}{d x^{2}}+V(x) u_{k}(x)=k^{2} u_{k}(x)
$$

and behave at infinity as

$$
\left(\frac{1}{2 \pi}\right)^{\frac{1}{2}}\left(e^{-i k x}+S(k) e^{i k x}\right)
$$

Let's apply this theorem to the problem considered above.

Using equations 42 and 43 we can write

$$
u_{k}(x)=-\left(\frac{1}{2 \pi}\right)^{\frac{1}{2}} e^{i \delta} C(k)^{-1} \sin (K x), \quad \text { for } x<a
$$

and

$$
u_{k}(x)=\left(\frac{1}{2 \pi}\right)^{\frac{1}{2}}\left(e^{-i k x}-e^{i 2 \delta} e^{i k x}\right), \quad \text { for } x>a,
$$

which is normalized according to the above theorem.

\subsection{The Titchmarsh-Weil m-coefficient (Everitt [1] )}

To normalize the eigenfunctions of the continuous spectrum of a problem that result from a potential like the one shown in Figure 2 the following method is easier. We mean easier in the sense that it does not require evaluation of divergent integrals but may be very laborious.

We rewrite equation (6) as

$$
\psi(x)=\sum c_{i} u_{E_{i}}(x)+\int C(E) u_{E}(x) \rho(E) d E .
$$

The function $\rho(E)$ is called spectral density and is related to the m-coefficient, that we shall define later in section 3.3.1 by the formula:

Recipe 1:

$$
\begin{aligned}
& \rho(E)=\frac{1}{2 \pi i} \lim _{\epsilon \rightarrow 0}[m(E+i \epsilon)-m(E-i \epsilon)] \\
& =\frac{1}{\pi} \lim _{\epsilon \rightarrow 0} \operatorname{Im}\{m(E+i \epsilon)\}
\end{aligned}
$$

where $\operatorname{Im}\{m(E+i \epsilon)\}$ means imaginary part of $m(E+i \epsilon)$.

In formula (48) the normalization of $u_{E}(x)$ is arbitrary but $\rho(E)$ must be calculated and it contains all the information we need about the continuous spectrum of the problem.

The normalization of $u_{E}(x)$ is obtained from equation (48) as follows

$$
\begin{aligned}
& \psi(x)=\sum c_{i} u_{E_{i}}(x)+\int\left(\int u_{E}^{*}(x) \psi(x) d x\right) \\
& \times u_{E}(x) \rho(E) d E,
\end{aligned}
$$

where we have replaced $C(E)$ in equation 49 by its value given by equation (8).

So

$$
\begin{aligned}
& \psi(x)=\sum c_{i} u_{E_{i}}(x)+\int\left(\int u_{E}^{*}(x) \rho^{1 / 2}(E) \psi(x) d x\right) \\
& \times u_{E}(x) \rho^{1 / 2}(E) d E .
\end{aligned}
$$

The function

$$
u_{E}(x) \rho^{1 / 2}(E)
$$

is properly normalized.

We shall illustrate now the method with examples. (An excellent example, the only one we could find in the literature dedicated to physics teaching, is given in reference [1].)

\subsubsection{How to calculate the m-coefficient}

We start with an operator given by its action (that is, what it does when acting on the function)

$$
-\frac{d^{2}}{d x^{2}}+V(x), \quad 0 \leq x<\infty
$$

and a boundary condition

$$
\psi^{\prime}(0)=\alpha \psi(0)
$$

For notational simplicity, we shall consider only the case where the operator has only continuous spectrum.

Given a function $f(x)$ we can write formula 48 as

$$
C(E)=\int_{0}^{\infty} f(x) u_{E}^{*}(x) d x
$$

and its inverse

$$
f(x)=\int_{0}^{\infty} C(E) u_{E}(x) \rho(E) d E,
$$

where $u_{E}(x)$ are eigenfunctions of the operator 53 , that is,

$$
-\frac{d^{2} u_{E}(x)}{d x^{2}}+V(x) u_{E}(x)=E u_{E}(x) .
$$

The normalization of $u_{E}(x)$ is arbitrary, but $\rho(E)$ must be calculated using equation 49 .

We now sketch how to calculate $m(E)$. 
First we calculate two eigenfunctions of the operator, $u_{E}(x)$ and $\gamma_{E}(x)$, with $\operatorname{Im}\{E\}>0$, such that

$$
u_{E}(0)=1, \quad u_{E}^{\prime}(0)=\alpha
$$

and

$$
\gamma_{E}(0)=0, \quad \gamma_{E}^{\prime}(0)=-1
$$

More generally we want two fundamental solutions of the equation. That is, $u_{E}(x)$ and $\gamma_{E}(x)$ must be linearly independent (Wronskian not zero) solutions of equation (57).

Recipe 2:

The m-coefficient is determined uniquely by the condition that $\gamma_{E}(x)+m(E) u_{E}(x)$ is square integrable, that is, belongs to $L^{2}(0 . \infty)$.

\subsubsection{Examples}

Example 5) As a first example of the procedure let's return to the Example 3 of section 3.1.

We want to find the normalization of the eigenfunctions of the operator

$$
-\frac{d^{2}}{d x^{2}} u_{k}(x)=k^{2} u_{k}(x)
$$

with boundary condition $u_{k}^{\prime}(0)=\alpha u_{k}(0)$. It is important to remember that $k^{2}$ is complex with $\operatorname{Im}\{k\}>0$.

Two fundamental solutions of equation $(60)$ are

$$
u_{k}(x)=\cos (k x)+\frac{\alpha}{k} \sin (k x)
$$

and

$$
\gamma_{k}(x)=-\frac{1}{k} \sin (k x)
$$

Note that the function 61 is an eigenvalue of equation (60) that satisfies the boundary condition $u_{k}^{\prime}(0)=$ $\alpha u_{k}(0)$.

The m-coefficient can then be easily determined

$$
\begin{aligned}
\gamma_{k}(x)+m(k) u_{k}(x) & =\left\{m(k)\left[\frac{1}{2}-\frac{\alpha}{2 i k}\right]+\frac{1}{2 i k}\right\} e^{-i k x} \\
& +\left\{m(k)\left[\frac{1}{2}+\frac{\alpha}{2 i k}\right]-\frac{1}{2 i k}\right\} e^{+i k x}
\end{aligned}
$$

This is square integrable if

$$
m(k)\left[\frac{1}{2}-\frac{\alpha}{2 i k}\right]+\frac{1}{2 i k}=0 .
$$

To see this, note that $\operatorname{Im}\{k\}>0$, therefore $\operatorname{Re}\left\{e^{-i k x}\right\}=$ $e^{-\operatorname{Im}\{k\} x}$ but $\operatorname{Re}\left\{e^{i k x}\right\}=e^{\operatorname{Im}\{k\} x}$ and so equation 64 has to be imposed. Re means real part.

From equation (64), we get

$$
m(k)=\frac{1}{\alpha-i k} .
$$

So that using equation 49

$$
\rho(k)=\frac{1}{\pi} \frac{k}{k^{2}+\alpha^{2}} .
$$

We now return to equation 50

$$
\psi(x)=\sum c_{i} u_{E_{i}}(x)+\int\left(\int u_{E}^{*}(x) \psi(x) d x\right) u_{E}(x) \rho(E) d E .
$$

Remembering that $E=k^{2}$ so that $d E=2 k d k$ and using equation 66 , we get

$$
\psi(x)=\left(\frac{2}{\pi}\right)^{\frac{1}{2}} k\left(\alpha^{2}+k^{2}\right)^{-\frac{1}{2}}\left(\cos (k x)+\frac{\alpha}{k} \sin (k x)\right) .
$$

This is the same as equation 28 .

Example 6) Normalize the functions of the Hamiltonian given by

$$
\left(-\frac{d^{2}}{d x^{2}}+V(x)\right) u_{E}(x)=E u_{E}(x)
$$

where the potential $V(x)$ is given by

$$
V(x)= \begin{cases}-V_{0}-F x, & 0<x<a \\ -F x, & x>a\end{cases}
$$

and the boundary condition $u_{E}(0)=0$.

This problem was completely solved in Dean and Fulling [1] or Titchmarsh [12, p. 92]. However here we describe only the solution for which $V_{0}=0$ and we follow Dean and Fulling [1].

First we put $V_{0}=0$ in equation 69 and change variables

$$
z=-F^{1 / 3} x-F^{-2 / 3} E
$$

and

$$
\psi(x)=\omega(z)
$$

Then equation

$$
-\frac{d^{2} \psi}{d x^{2}}-F x \psi=E \psi
$$

with boundary condition $\psi(0)=0$ becomes

$$
\frac{d^{2} \omega}{d z^{2}}-z \omega=0
$$

which is the Airy's equation 13 .

The Airy's equation has two linearly independet solutions: $\operatorname{Ai}(z)$ and $\operatorname{Bi}(z)$.

We need to find combinations of $\operatorname{Ai}(z)$ and $\operatorname{Bi}(z)$ that satisfy (58) with $\alpha=1$ and (59). Write them as

$$
\begin{aligned}
& u_{E}(x)=a \operatorname{Ai}(z)+b \operatorname{Bi}(z) \\
& \gamma_{E}(x)=c \operatorname{Ai}(z)+d \operatorname{Bi}(z) .
\end{aligned}
$$


Now we invert (74) and $(75)$ to write

$$
\begin{aligned}
& \operatorname{Ai}(z)=e u_{E}(x)+f \gamma_{E}(x) \\
& \operatorname{Bi}(z)=g u_{E}(x)+h \gamma_{E}(x)
\end{aligned}
$$

where

$$
\begin{aligned}
e & =-F^{1 / 3}(\mathrm{Ai})^{\prime}\left(-F^{-2 / 3} E\right), \\
f & =\operatorname{Ai}\left(-F^{-2 / 3} E\right), \\
g & =-F^{1 / 3}(\mathrm{Bi})^{\prime}\left(-F^{-2 / 3} E\right), \\
h & =\operatorname{Bi}\left(-F^{-2 / 3} E\right) .
\end{aligned}
$$

Taking advantage of the fact that the Wronskian of the Airy function is $W[\operatorname{Ai}(z), \operatorname{Bi}(z)]=\pi^{-1}$, we calculate

$$
\begin{aligned}
& a=\pi F^{-1 / 3} \operatorname{Bi}\left(-F^{-2 / 3} E\right), \\
& b=-\pi F^{-1 / 3} \operatorname{Ai}\left(-F^{-2 / 3} E\right), \\
& c=\pi(\mathrm{Bi})^{\prime}\left(-F^{-2 / 3} E\right), \\
& d=-\pi(\mathrm{Ai})^{\prime}\left(-F^{-2 / 3} E\right) .
\end{aligned}
$$

We now have two functions $u_{E}(x)$ and $\gamma_{E}(x)$ that satisfy the differential equation $\sqrt{72}$ and the boundary conditions (58) and (59).

We now must find a linear combination of these solutions

$$
\gamma_{E}(x)+m(E) u_{E}(x)
$$

that is square integrable.

This linear combination found by asymptotic analysis (see Dean and Fulling [1]) is

$\gamma_{E}(x)+\frac{i(\mathrm{Bi})^{\prime}\left(-F^{-2 / 3} E\right)-(\mathrm{Ai})^{\prime}\left(-F^{-2 / 3} E\right)}{-i \operatorname{Bi}\left(-F^{-2 / 3} E\right)+\operatorname{Ai}\left(-F^{-2 / 3} E\right)} F^{1 / 3} u_{E}(x)$.

Readers unfamiliar with asymptotic analysis should consult Wasow [14, Olver [15, p.54] or Mazzitelli et al. 16.

Hence, by Recipe 2,

$$
m(E)=\frac{i(\mathrm{Bi})^{\prime}\left(-F^{-2 / 3} E\right)-(\mathrm{Ai})^{\prime}\left(-F^{-2 / 3} E\right)}{-i \operatorname{Bi}\left(-F^{-2 / 3} E\right)+\operatorname{Ai}\left(-F^{-2 / 3} E\right)} F^{1 / 3},
$$

and, by Recipe 1 ,

$\rho(E)=F^{1 / 3} \pi^{-2} /\left[\mathrm{Ai}^{2}\left(-F^{-2 / 3} E\right)+\mathrm{Bi}^{2}\left(-F^{-2 / 3} E\right)\right]$.

By replacing in equation $(52) u_{E}(x)$ given by equation (74) and $\rho^{1 / 2}(E)$ given by the square root of equation (89), we get

$$
\begin{aligned}
& u_{E}(x)=\left\{\pi F^{-1 / 3} \operatorname{Bi}\left(-F^{-2 / 3} E\right) \operatorname{Ai}\left(-F^{1 / 3} x-F^{-2 / 3} E\right)+\right. \\
& \left.-\pi F^{-1 / 3} \operatorname{Ai}\left(-F^{-2 / 3} E\right) \operatorname{Bi}\left(-F^{1 / 3} x-F^{-2 / 3} E\right)\right\} \times \\
& \times \frac{F^{1 / 6}}{\pi}\left[\mathrm{Ai}^{2}\left(-F^{-2 / 3} E\right)+\mathrm{Bi}^{2}\left(-F^{-2 / 3} E\right)\right]^{-1 / 2},
\end{aligned}
$$

which completes the solution.
Remark 2 Divergent integrals and the delta function

In section 3.1 we considered the following integral

$$
\int_{0}^{\infty} \sin \left(k_{1} x\right) \sin \left(k_{2} x\right) d x
$$

which in the usual sense is at least dubious. We want to show that it is in fact equal to $\frac{\pi}{2} \delta\left(k_{1}-k_{2}\right)$.

The symbol $\delta\left(k_{1}-k_{2}\right)$ is a distribution in this case the so-called Dirac Delta Function. A distribution is a linear functional that when acting on a good function produces a number. A simple example of a functional is one generated by a function $F(x)$. In fact, given any good function $f(x)$, this functional is given by

$$
\int_{-\infty}^{\infty} F(x) f(x)=\text { Number } .
$$

A delta function is a functional that acts in a function as follows.

$$
\int_{-\infty}^{\infty} \delta(y-x) f(x)=f(y)
$$

Note that there is no ordinary function that generates this functional.

So, we want to show that the mathematical object $\int_{0}^{\infty} \sin \left(k_{1} x\right) \sin \left(k_{2} x\right) d x$ is a delta function.

That is, we want to show that

$$
\begin{aligned}
& \frac{2}{\pi} \int_{0}^{\infty} \sin \left(k_{1} x\right) \sin \left(k_{2} x\right) d x \\
& =\lim _{L \rightarrow \infty} \frac{2}{\pi} \int_{0}^{L} \sin \left(k_{1} x\right) \sin \left(k_{2} x\right) d x
\end{aligned}
$$

behaves as

$$
\frac{2}{\pi} \int_{0}^{\infty} \lim _{L \rightarrow \infty} \int_{0}^{L} \sin \left(k_{1} x\right) \sin \left(k_{2} x\right) d x f\left(k_{1}\right)=f\left(k_{2}\right) .
$$

The manipulations carried out in section 3.1 show that

$$
\frac{2}{\pi} \int_{0}^{\infty} \sin \left(k_{1} x\right) \sin \left(k_{2} x\right) d x=\delta\left(k_{1}-k_{2}\right) .
$$

Another approach to obtain the $\delta$ function can be used, and this was probably noticed by attentive readers. In this approach, the $\delta$ function, $\delta(x)$, is defined as limits of sequences that approach a function that is 0 if $x \neq 0$ and $\infty$ if $x=0$.

One such sequence is

$$
\lim _{a \rightarrow \infty} \frac{\sin (a x)}{a}=\pi \delta(x) .
$$

Note that, when $k_{1} \rightarrow k_{2}$, equation 37 becomes

$$
\frac{1}{2} C^{*}\left(k_{1}\right) C\left(k_{2}\right)\left\{\frac{1}{k_{1}-k_{2}} \sin \left[\left(k_{1}-k_{2}\right) R\right]\right\}
$$


and, therefore, taking the limit as $R \rightarrow \infty$ we get from equation 97)

$$
\frac{1}{2}\left|C\left(k_{1}\right)\right|^{2} \delta\left(k_{1}-k_{2}\right)
$$

Many other sequences that tend to a $\delta$ function can be found in [2, p.103] and the whole approach is explained in 17 .

\section{Acknowledgements}

The authors acknowledge partial support from CNPq.

\section{References}

[1] C.L. Dean and S.A. Fulling, Am. J. Phys. 50, 540 (1982).

[2] W. Greiner Quantum Mechanics: An Introduction (Springer, Berlin, 1994), $3^{a}$ ed.

[3] B. Friedman, Principles and Techniques of Applied Mathematics (John Wiley and Sons, New York, 1956).

[4] J. Audretsch, U. Jasper and V.D. Skarzhinsky, J. Phys. A: Math. Gen. 28, 2359 (1995).

[5] V.S. Araujo, F.A.B. Coutinho and J.F. Perez, Braz. J. Phys. 32, 636 (2002).

[6] R.P. Kanwal, Generalized functions. Theory and Applications (Birkhauser, Boston, 2004), $3^{a}$ ed.

[7] K.R. Brownstein, Am. J. Phys. 43, 173 (1975).

[8] T.M. Apostol, Mathematical Analysis (Addison-Wesley, Menlo Park, 1974), $2^{a}$ ed.

[9] R.V. Churchill, Operational Mathematics (McGraw-Hill, New York, 1958)

[10] A.M. Perelomov and Y.B. Zel'dovich, Quantum Mechanics - Selected Topics (World Scientific, Singapore, 1998).

[11] W.N. Everitt, J. Comput. Appl. Math. 171, 185 (2004).

[12] E.C. Titchmarsh, Eigenfunctions Expansions Associated with Second-Order Differential Equations (Oxford University Press, London, 1962).

[13] M. Abramowitz and I.A. Stegun, Handbook of Mathematical Functions (Dover, New York, 1965).

[14] W. Wasow, Asymptotic Expansions for Ordinary Differential Equations (Wiley, New York, 1965).

[15] F.W.J. Olver, Asymptotics and Special Functions (A K Peters, Wellesley, 1997).

[16] F.D. Mazzitelli, M.D. Mazzitelli and P.I. Soubelet, Am. J. Phys. 85, 750 (2017).

[17] M.J. Lighthill, Introduction to Fourier Analysis and Generalised Functions (Cambridge University Press, London, 1964). 